\title{
The Higher Order Lifts on the Extended Vector Bundles
}

\author{
Cansel Yormaz ${ }^{1}$, Ali Görgülï̈ ${ }^{2}$ \\ Department of Mathematics, Faculty of Science and Art, Pamukkale University, Denizli-Turkey ${ }^{1}$ \\ Department of Mathematics, Faculty of Science and Art, Osmangazi University, Eskişehir-Turkey²
}

\begin{abstract}
In this study, the higher order vertical and complete lifts of the tensor fields, which are defined on the extended vector bundles has been obtained.
\end{abstract}

Keywords: The extended vector bundles, The vertical and complete lifts of the tensor fields.

\section{INTRODUCTION}

In the previous studies [1-5], [11], [12] the first order lifts of the differential elements defined on a manifold $\mathrm{M}$ to its tangent bundle TM in the differential geometry have been obtained. Furthermore in the studies of [3] and [5], the second order lifts of the differential elements defined on a manifold $\mathrm{M}$ to its the second order tangent bundle TTM have been calculated. the structure of extended manifolds had been obtained. Especially, in the studies of [7], the second order lifts of the differential elements defined on a manifold $\mathrm{M}$ to its the second canonical extended manifold ${ }^{2} \mathrm{M}$ are derived.

In $\S 2$ of this paper, we define the vertical and complete lifts of higher order of functions defined on the vector bundle $\pi=(\mathrm{E}, \pi, \mathrm{M})$ to its the extended vector bundles $\pi$ $\mathrm{k}=\left({ }^{\mathrm{k}} \mathrm{E}, \pi^{\mathrm{k}},{ }^{\mathrm{M}}\right)[2]$.

We study in more detail the vertical and complete lifts of vector fields defined on the vector bundle $\pi$ to its the extended vector bundles $\pi^{\mathrm{k}}$ by considering the lifts of functions on the extended vector bundle $\pi^{\mathrm{k}}$ in the $\S 2$.

In the $\S 4$, we calculate the vertical and complete lifts of 1 -forms defined on the vector bundle $\pi$ to its the extended vector bundles $\pi^{\mathrm{k}}$ by considering the lifts of vector fields on the extended vector bundle $\pi^{\mathrm{k}}$ in the $\S 2$.

In the $\S 4$, we compare the vertical and complete lifts of differential elements in the previous studies with the vertical and complete lifts of differential elements in this study. In this paper, all manifolds and mappings are assumed to be differentiable of class $\mathrm{C}^{\infty}$, unless otherwise stated and the sum is taken over repeated indices.

\section{THE LIFTS OF FUNCTIONS}

Let $\mathrm{f}: \mathrm{E} \rightarrow \mathbf{R}$ be a function defined on the vector bundle $\pi=(E, \pi, M)$. if we define the vertical lift of a function $\mathrm{f}$ defined on the vector bundle $\pi$ to its the extended vector bundle $\pi^{\mathrm{k}}=\left({ }^{\mathrm{k}} \mathrm{E}, \pi^{\mathrm{k}},{ }_{\mathrm{k}} \mathrm{M}\right)$ using the induction method for integer number $\mathrm{k}$, then:

DEFINITION 2.1. Let $\mathrm{f}: \mathrm{E} \rightarrow \mathbf{R}$ be a function defined on the vector bundle $\pi$ then it is called the vertical lift of order $\mathbf{k}$ of $f$ to the extended vector bundle $T \pi^{k-1}$ which a function $\mathrm{f}^{\mathrm{V}}$ defined on the vector bundle $\mathrm{T} \pi$ $\mathrm{k}-1$ defined with below the equation

$$
\mathrm{f}^{\mathrm{V}^{\mathrm{k}}}=\mathrm{f} \text { o } \tau_{0} \mathrm{E}^{\mathrm{o} \tau 1_{\mathrm{E}}} \mathrm{o \ldots o} \tau \mathrm{k}-1_{\mathrm{E}} \text {. }
$$

For all the tangent vector $\mathrm{H} \in \mathrm{T}\left({ }^{\mathrm{k}-1} \mathrm{E}\right)$ and ${ }^{\tau 0} \mathrm{E}^{\tau 1} \mathrm{E} \ldots \tau$ $k-1 E^{(H)}=e \in{ }^{0} E ;$

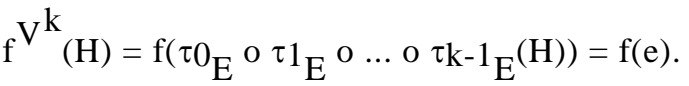

DEFINITION 2.2. Let $\mathrm{f}^{\mathrm{V}}$ be vertical lift of $\mathrm{f}$ to $\mathrm{T} \pi^{\mathrm{k}-}$ 1 . Then $\pi^{\mathrm{k}}$ is the subvector bundle of $\mathrm{T} \pi^{\mathrm{k}-1}$ and ${ }^{\mathrm{k}} \mathrm{E}$ is the subset of $\mathrm{T}\left({ }^{\mathrm{k}-1} \mathrm{E}\right)$, therefore if we restrict the vertical lift $\mathrm{f}^{\mathrm{V}}$ to $\mathrm{k}_{\mathrm{E}}$, then the function $\left.\mathrm{f}^{\mathrm{k}}\right|_{\mathrm{E}}$ is defined on the extended vector bundle $\pi^{\mathrm{k}}$ and it is called the vertical lift of order $k$ of $f$ to the extended vector bundle $\pi^{\mathrm{k}}$ which it is a function defined on the vector bundle $\mathrm{T} \pi^{\mathrm{k}-1}$ defined with below the equation

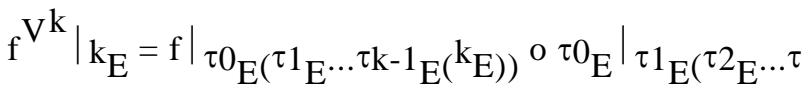

$$
\begin{aligned}
& \left.\mathrm{k}-1_{\mathrm{E}}\left({ }^{\mathrm{k}} \mathrm{E}\right)\right) \text { o .. o } \tau_{\mathrm{k}-1_{\mathrm{E}}} \mid \mathrm{k}_{\mathrm{E}} \text { (2.2) }
\end{aligned}
$$


For shortness, we denote $f_{k}^{V^{k}}$ with $\left.\mathrm{f}^{\mathrm{V}^{\mathrm{k}}}\right|_{\mathrm{k}_{\mathrm{E}}}$. In the complete lift $\mathrm{f}^{\mathrm{C}}$ to the extended manifold ${ }^{1} \mathrm{E}$; then that case; for all tangent vector $\mathrm{H} \in{ }^{\mathrm{k}} \mathrm{E} \subset \mathrm{T}\left({ }^{\mathrm{k}-1} \mathrm{E}\right)$ and $\left.\mathrm{f}^{\mathrm{C}}\right|_{1_{\mathrm{E}}}=\left.\mathrm{f}^{\mathrm{C}}\right|_{\mathrm{TE}}=\left(\partial_{0 \mathrm{i}} \mathrm{f}\right)^{\mathrm{V}}\left|{ }_{1_{\mathrm{E}}} \mathrm{x}^{1 \mathrm{i}}+\left(\partial_{0 \alpha} \mathrm{f}\right)^{\mathrm{V}}\right|{ }_{1_{\mathrm{E}}} \mathrm{u}^{1}$ ${ }^{\tau 0} \mathrm{E}^{\tau 1} \mathrm{E} \cdots \tau_{\mathrm{k}-1} \mathrm{E}^{(\mathrm{H})}=\mathrm{e} \in{ }^{0} \mathrm{E}$

$$
\alpha=\left(\partial_{0 \mathrm{i}} \mathrm{f}\right)_{1}^{V^{1}} \mathrm{x}^{1 \mathrm{i}}+\left(\partial_{0 \alpha^{\mathrm{f}}}\right)_{1}^{V^{1}} \mathrm{u}^{1 \alpha} \text {. }
$$

$f_{k}^{V^{k}}(\mathrm{H})=\mathrm{f}^{\mathrm{V}^{\mathrm{k}}}(\mathrm{H})=\mathrm{f}\left(\tau 0_{\mathrm{E}}\right.$ o $\tau 1_{\mathrm{E}}$ o $\ldots$ o $\left.\tau_{\mathrm{k}-1} \mathrm{E}_{\mathrm{E}}(\mathrm{H})\right)=\left.\mathrm{f}^{\mathrm{C}}\right|_{1_{\mathrm{E}}}$ is called the complete lift of function $\mathrm{f}$ in $\pi$ to $\mathrm{f}(\mathrm{e})$. the extended vector bundle $\pi^{1}$.

PROPOSITION 2.1. For all the functions $f, g \in \mathfrak{I}_{0}{ }^{0}(\pi)$ In addition to for all tangent vector $\mathrm{Z} \in{ }^{1} \mathrm{E}=\mathrm{TE}$ and $\tau$ and integer numbers $0 \leq \mathrm{r} \leq \mathrm{k}$;

$$
\begin{aligned}
& \text { i) }(\mathrm{f}+\mathrm{g})_{r}^{V^{r}}=\mathrm{f}_{r}^{V^{r}}+\mathrm{g}_{r}^{V^{r}} \\
& \text { ii) }(\mathrm{f} . \mathrm{g})_{r}^{V^{r}}=\mathrm{f}_{r}^{V^{r}} \cdot \mathrm{g}_{r}^{V^{r}}
\end{aligned}
$$

Furthermore; using by the induction method, we define the complete lift of order $\mathrm{k}$ defined on its the extended vector bundle $\pi^{\mathrm{k}}$. Let $\mathrm{f}$ be a function defined on the vector bundle $\pi$. Then the differential of $\mathrm{f}$ is defined by

$$
\mathrm{df}=\frac{\partial f}{\partial x^{0 i}} \mathrm{dx}^{0 \mathrm{i}}+\frac{\partial f}{\partial u^{0 \alpha}} \mathrm{du}^{0 \alpha}
$$

with respect to the adapted coordinates $\left(\mathrm{x}^{0 \mathrm{i}}, \mathrm{u}^{0 \alpha}\right)$ in $\mathrm{E}$.

Thus if we define the linear map

$\mathfrak{\imath}_{1}: \mathfrak{I}_{1}^{0}\left(\pi^{0}\right) \rightarrow \mathfrak{I}_{0}^{0}\left(\mathrm{~T} \pi^{0}\right)$

${ }^{0} \mathrm{E}^{(\mathrm{Z})}=\mathrm{e} \in{ }^{0} \mathrm{E}$;

$$
f_{1}^{C^{1}}(\mathrm{Z})=\left(\partial_{0 \mathrm{i}} \mathrm{f}\right)_{1}^{V^{1}}(\mathrm{Z}) \mathrm{x}^{1 \mathrm{i}}(\mathrm{Z})+\left(\partial_{0 \alpha} \mathrm{f}\right)_{1}^{V^{1}}(\mathrm{Z}) \mathrm{u}^{1 \alpha}
$$

$$
=\left(\partial_{0 i^{f}}\right)(e) Z\left[x^{0 i}\right]+\left(\partial_{0 \alpha} f\right)(e) Z\left[u^{0 \alpha}\right] .
$$

Thus we define the complete lift of order $\mathrm{k}$ of $\mathrm{f}$ in $\pi$ to the vector bundle $\pi^{\mathrm{k}}$. Let $\bar{f}:{ }^{k-1} E \rightarrow R$ be a function defined on the vector bundle $\pi^{\mathrm{k}-1}$. Then the differential of $\bar{f}$ is defined by

$$
\mathrm{d} \bar{f}=\frac{\partial \bar{f}}{\partial x^{r i}} \mathrm{dx}{ }^{\mathrm{ri}}+\frac{\partial \bar{f}}{\partial u^{r \alpha}} \mathrm{du}^{\mathrm{r} \alpha}
$$

with respect to the adapted coordinates $\left(\mathrm{x}^{\mathrm{ri}}, \mathrm{u}^{\mathrm{r} \alpha}\right) 0 \leq \mathrm{r} \leq$ $\mathrm{k}-1$ in ${ }^{\mathrm{k}-1} \mathrm{E}$.

If we define the linear map

with respect to $\operatorname{Sp}\left\{\mathrm{dx}^{0 \mathrm{i}}, \mathrm{du}^{0 \alpha}: 1 \leq \mathrm{i} \leq \mathrm{m}, 1 \leq \alpha \leq \mathrm{n}\right\}=\mathfrak{\Im}_{1}{ }^{0}$ ( $\left.\pi^{0}\right)$ with $\mathrm{\imath}_{1}\left(\mathrm{dx}^{0 \mathrm{i}}\right)=\dot{x}^{0 \mathrm{i}}, \iota_{1}\left(\mathrm{du}^{0 \alpha}\right)=\dot{u}^{0 \alpha}$

DEFINITION 2.3. Let $\mathrm{f}: \mathrm{E} \rightarrow \mathbf{R}$ be a function defined on the vector bundle $\pi$ and the differential $\mathrm{df}$ of $\mathrm{f}$ be in form (2.3). Thus we write $\mathrm{f}^{\mathrm{C}}$ for the function in the vector bundle $\mathrm{T} \pi^{0}$ defined by

$$
\mathrm{f}^{\mathrm{C}}=\mathrm{r}_{1}(\mathrm{df})
$$

and call $\mathrm{f}^{\mathrm{C}}$ the complete lift of function $\mathrm{f}$ in $\pi$ to vector bundle $\mathrm{T} \pi^{0}$. The complete lift $\mathrm{f}^{\mathrm{C}}$ has the local expression

$$
\mathrm{f}^{\mathrm{C}}={ }_{1}(\mathrm{df})=\left(\partial_{0 \mathrm{f}} \mathrm{f}\right)^{\mathrm{V}} \dot{x}^{0 \mathrm{i}}+\left(\partial_{0 \alpha} \mathrm{f}\right)^{\mathrm{V}} \dot{u} 0 \alpha
$$
$\mathfrak{l}_{\mathrm{k}}: \mathfrak{I}_{1}^{0}\left(\pi^{\mathrm{k}-1}\right) \rightarrow \mathfrak{I}_{0}^{0}\left(\mathrm{~T} \pi^{\mathrm{k}-1}\right)$

with respect to $\operatorname{Sp}\left\{\mathrm{dx}^{\mathrm{ri}}, \mathrm{du}^{\mathrm{r} \alpha}: 0 \leq \mathrm{r} \leq \mathrm{k}-1\right\}=\mathfrak{\Im}_{1}{ }^{0}\left(\mathrm{~T} \pi^{\mathrm{k}-1}\right)$ with

$\mathrm{t}_{\mathrm{k}}\left(\mathrm{dx}{ }^{\mathrm{ri}}\right)=\dot{x}^{\mathrm{ri}}, \quad \mathrm{t}_{\mathrm{k}}\left(\mathrm{du}{ }^{\mathrm{r} \alpha}\right)=\dot{u}^{\mathrm{r} \alpha} \quad 0 \leq \mathrm{r} \leq \mathrm{k}-1$

DEFINITION 2.4. Let $\bar{f}$ be a function defined on the vector bundle $\pi^{\mathrm{k}-1}$ and the differential $\mathrm{d} \bar{f}$ of $\bar{f}$ be in form (2.7). Thus we write $\bar{f}^{\mathrm{C}}$ for the function in the vector bundle $\mathrm{T} \pi^{\mathrm{k}-1}$ defined by

$$
\bar{f}^{\mathrm{C}}=\mathrm{l}_{\mathrm{k}}(\mathrm{d} \bar{f})
$$

and call $\bar{f}^{\mathrm{C}}$ the complete lift of function $\bar{f}$ in $\pi$ to vector bundle $\mathrm{T} \pi^{\mathrm{k}-1}$. The complete lift $\bar{f}^{\mathrm{C}}$ has the local expression

with respect to adapted coordinates $\left(\mathrm{x}^{0 \mathrm{i}}{ }_{, \mathrm{u}}{ }^{0 \alpha}, \dot{x}^{0 \mathrm{i}}, \dot{u}^{0 \alpha}\right)$ in $\mathrm{T} \pi$, where $\partial_{0 \mathrm{i}} \mathrm{f}$ and $\partial_{0 \alpha} \mathrm{f}$ denotes $\frac{\partial f}{\partial x^{0 i}}$ and $\frac{\partial f}{\partial u^{0 \alpha}}$ $\bar{f}^{\mathrm{C}}=\mathrm{v}_{\mathrm{k}}(\mathrm{d} \bar{f})=\left(\partial_{\mathrm{ri}} \bar{f}\right) \mathrm{V} \dot{x}^{\mathrm{ri}}+\left(\partial_{\mathrm{r} \alpha} \bar{f}\right)^{\mathrm{V}} \dot{u}^{\mathrm{r} \alpha}(2.9)$ respectively. Therefore $\pi^{1}$ equal to $\mathrm{T} \pi$; if we restrict a) $0 \leq \mathrm{r} \leq \mathrm{k}-1$ in $\mathrm{T} \pi^{\mathrm{k}-1}$. 
Vol. 5, Issue 12, December 2016

Let $\mathrm{f}$ be a function defined on the vector bundle $\pi$ and the complete lift of order k-1 $f_{k-1}^{C^{k-1}}$ of $\mathrm{f}$ to the extended vector bundle $\pi^{\mathrm{k}-1}$. Then, therefore the complete lift of order $\mathrm{k}-1 f_{k-1}^{C^{k-1}}$ of $\mathrm{f}$ is defined on $\pi$ $\mathrm{k}-1$, if we take $\bar{f}$ equal to $f_{k-1}^{C^{k-1}}$ and we write in (2.9) then we obtain

$$
\begin{gathered}
\left(f_{k-1}^{C^{k-1}}\right)^{\mathrm{C}}=\mathrm{l}_{\mathrm{k}}\left(\mathrm{d} f_{k-1}^{C^{k-1}}\right)=\left(\partial_{\mathrm{ri}} f_{k-1}^{C^{k-1}}\right)^{\mathrm{V}} \dot{x}^{\mathrm{ri}}+(\partial \\
\left.{ }_{\mathrm{r} \alpha} f_{k-1}^{C^{k-1}}\right)^{\mathrm{V}} \dot{u}^{\mathrm{r} \alpha} .
\end{gathered}
$$

Therefore, $\pi^{\mathrm{k}}$ is subvector bundle of $\mathrm{T} \pi^{\mathrm{k}-1}$ and ${ }^{\mathrm{k}} \mathrm{E}$ is submanifold of $\mathrm{T}\left({ }^{\mathrm{k}-1} \mathrm{E}\right)$; we restrict the complete lift ( $\left.f_{k-1}^{C^{k-1}}\right)^{\mathrm{C}}$ to the total space ${ }^{\mathrm{k}} \mathrm{E}$ of $\mathrm{T} \pi^{\mathrm{k}-1}$ and we consider the adapted coordinates $\left(\dot{x}^{\mathrm{ri}}, \dot{u}^{\mathrm{r} \alpha}\right)=\left(\mathrm{x}^{\mathrm{ri}}, \mathrm{u}^{\mathrm{r} \alpha}\right)$ for $0 \leq \mathrm{r} \leq \mathrm{k}-2$ on the extended manifold ${ }^{\mathrm{k}} \mathrm{E}$ from the defining condition of ${ }^{\mathrm{k}} \mathrm{E}$, then, we get

$$
\begin{aligned}
& \left.\left(f_{k-1}^{C^{k-1}}\right)^{\mathrm{C}}\right|_{\mathrm{k}_{\mathrm{E}}}=f_{k}^{C^{k}}=\left.\left(\partial_{\mathrm{ri}} f_{k-1}^{C^{k-1}}\right)^{\mathrm{V}}\right|_{\mathrm{k}_{\mathrm{E}}} \mathrm{x}^{\mathrm{r}+1 \mathrm{i}}+( \\
& \left.\partial_{\mathrm{r} \alpha} f_{k-1}^{C^{k-1}}\right)\left.^{\mathrm{V}}\right|_{\mathrm{k}_{\mathrm{E}}} \mathrm{u}^{\mathrm{r}+1 \alpha} \text {. }
\end{aligned}
$$

For all tangent vector $\mathrm{H} \in{ }^{\mathrm{k}} \mathrm{E} \subset \mathrm{T}\left({ }^{\mathrm{k}-1} \mathrm{E}\right)$ and $1_{\mathrm{E}}(\mathrm{H})=\mathrm{G} \in{ }^{\mathrm{k}-1} \mathrm{E}$;

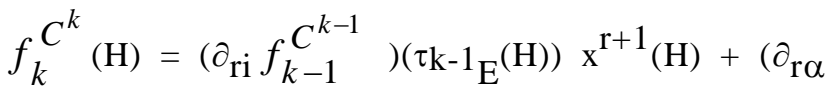
$\left.f_{k-1}^{C^{k-1}}\right)\left(\tau_{\mathrm{k}-1} \mathrm{E}^{(\mathrm{H}))} \mathrm{u}^{\mathrm{r}+1 \alpha_{(\mathrm{H})}}\right.$

$=\left(\partial_{\mathrm{ri}} f_{k-1}^{C^{k-1}}\right)(\mathrm{G}) \mathrm{H}\left[\mathrm{x}^{\mathrm{ri}}\right]+\left(\partial_{\mathrm{r} \alpha} f_{k-1}^{C^{k-1}}\right)(\mathrm{H}) \mathrm{H}\left[\mathrm{u}^{\mathrm{r} \alpha}\right]$.

DEFINITION 2.5. Let $\mathrm{f}$ be a function defined on the vector bundle $\pi$. Then it is called the complete lift of order $\mathbf{k} f_{k}^{C^{k}}$ of the function $\mathrm{f}$ in $\pi$ to the extended vector bundle $\pi^{\mathrm{k}}$ that it is defined with equation (2.11).

The complete-vertical lift of order $(r, s)$ of a function $f$ defined on the vector bundle $\pi$ to its the extended vector bundle $\pi^{\mathrm{k}}$ can be defined.

For the integer numbers $0 \leq \mathrm{r} \leq \mathrm{k}$ and $0 \leq \mathrm{s} \leq \mathrm{k}-\mathrm{r}$, we suppose that $f_{r}^{C^{r}}$ is the complete lift of order $\mathrm{r}$ of the function $\mathrm{f}$ in $\pi$ to its the extended vector bundle $\pi^{\mathrm{k}}$. In that case, we write

$$
\begin{aligned}
& \left(f_{r}^{C^{r}}\right)_{s}^{V^{s}}=f_{r+s}^{C^{r} V^{s}}=f_{k}^{C^{r} V^{s}}=\left.f_{r}^{C^{r}}\right|_{\tau_{\mathrm{r}}}\left({ }^{\tau} \mathrm{r}+1_{\mathrm{E}} \ldots \tau\right.
\end{aligned}
$$

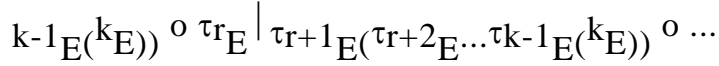

$$
\begin{aligned}
& \text {... o } \tau_{\mathrm{k}-1} \mathrm{E}_{\mathrm{E}} \mid \mathrm{k}_{\mathrm{E}} \text { (2.12) }
\end{aligned}
$$

DEFINITION 2.6. Let $\mathrm{f}$ be a function defined on the vector bundle $\pi$. Then it is called the completevertical lift of order $(\mathbf{r}, \mathbf{s}) f_{k}^{C^{r} V^{s}}$ of the function $\mathrm{f}$ in $\pi$ to its the extended vector bundle $\pi^{\mathrm{k}}$ that it is defined with equation (2.12).

For all tangent vector $\mathrm{H} \in{ }^{\mathrm{k}} \mathrm{E} \subset \mathrm{T}\left({ }^{\mathrm{k}-1} \mathrm{E}\right)$ and ${ }^{{ }_{\mathrm{r}}} \mathrm{E}^{\tau_{\mathrm{r}+1}} \mathrm{E}^{\cdots}$

$$
\tau_{\mathrm{k}-1}{ }_{\mathrm{E}}(\mathrm{H})=\mathrm{B} \in{ }^{\mathrm{r}} \mathrm{E}
$$

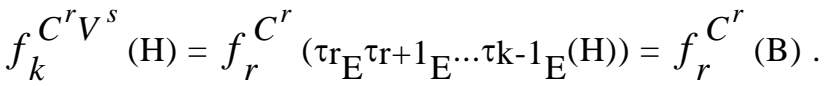

REMARK 2.1. The complete-vertical lift of order $(r, s)$ of a function defined on the vector bundle $\pi$ to $\pi^{\mathrm{k}}$ is equal to vertical-complete lift of order $(s, r)$ of a function defined on the vector bundle $\pi$ to $\pi^{\mathrm{k}-1}$. That is $f_{k}^{C^{r} V^{s}}=f_{k}^{V^{s} C^{r}}$.

PROPOSITION 2.2. For all the functions $f, g \in \mathfrak{I}_{0}{ }^{0}(\square)$ and integer numbers $0 \leq \mathrm{r} \leq \mathrm{k}$;

i) $(\mathrm{f}+\mathrm{g}){ }_{r}^{C^{r}}=\mathrm{f}_{r}^{C^{r}}+\mathrm{g}_{r}^{C^{r}}$

ii) (f.g ) ${ }_{r}^{C^{r}}=\sum_{i=0}^{r}\left(\begin{array}{l}r \\ i\end{array}\right) f_{r}^{C^{r-i} V^{i}} g_{r}^{C^{i} V^{r-i}}$

iii) $\left(\frac{\partial f}{\partial x^{0 i}}\right)_{r}^{C^{r}}=\frac{\partial f_{r}^{C^{r}}}{\partial x^{0 i}},\left(\frac{\partial f}{\partial u^{0 \alpha}}\right)_{r}^{C^{r}}=\frac{\partial f_{r}^{C^{r}}}{\partial u^{0 \alpha}}$

iv) $\left(\frac{\partial f}{\partial x^{0 i}}\right)_{r}^{V^{r}}=\frac{\partial f_{r}^{C^{r}}}{\partial x^{r i}},\left(\frac{\partial f}{\partial u^{0 \alpha}}\right)_{r}^{V^{r}}=\frac{\partial f_{r}^{C^{r}}}{\partial u^{r \alpha}}$

v) $\left(\frac{\partial f}{\partial x^{0 i}}\right){ }_{k}^{C^{k-r} V^{r}}=\frac{\partial f_{k}^{C^{k}}}{\partial x^{r i}},\left(\frac{\partial f}{\partial u^{0 \alpha}}\right)_{k}^{C^{k-r} V^{r}}=$ $\frac{\partial f_{k}^{C^{k}}}{\partial u^{r \alpha}}$

\section{THE LIFTS OF VECTOR FIELDS}

Let $X$ be a vector field defined on the vector bundle and local expression of $\mathrm{X}$ 


$$
\mathrm{X}=\mathrm{X}^{0 \mathrm{i}} \frac{\partial}{\partial x^{0 i}}+\mathrm{X}^{0 \alpha} \frac{\partial}{\partial u^{0 \alpha}}
$$

Thus if we define the vertical lift of a vector field $X$ defined on the vector bundle $\pi$ to its the extended vector bundle $\pi^{\mathrm{k}}=\left({ }^{\mathrm{k}} \mathrm{E}, \pi^{\mathrm{k}},{ }^{\mathrm{k}} \mathrm{M}\right)$ using the induction method for integer number $\mathrm{k}$, then:

DEFINITION 3.1. Let $X$ be a vector field defined on the vector bundle $\square$. In that case; the vertical lift of order $\mathbf{k}$ of $\mathbf{X}$ to the extended vector bundle $\pi^{\mathrm{k}}$ is a vector field defined on $\pi^{\mathrm{k}}$ which it is defined with the below equation

$$
\mathrm{X}_{k}^{V^{k}}\left(\mathrm{f}_{k}^{C^{k}}\right)=(\mathrm{X}[\mathrm{f}])_{k}^{V^{k}} ; \quad \forall \mathrm{f} \in \mathfrak{I}_{0}^{0}(\square) .
$$

Now we give the below theorem connected with the components of the vertical lift of order $\mathrm{k}$ of $\mathrm{X}$.

PROPOSITION 3.1. Let $X$ be a vector field defined on the vector bundle $\square$ with form (3.1). In that case; the local expression of the vertical lift $\mathrm{X}_{k}^{V^{k}}$ of order $\mathrm{k}$ of $\mathrm{X}$ to the extended vector bundle $\pi^{\mathrm{k}}$ is

$$
X_{k}^{V^{k}}=\left(X^{o i}\right)_{k}^{V^{k}} \frac{\partial}{\partial x^{k i}}+\left(X^{o \alpha}\right)_{k}^{V^{k}} \frac{\partial}{\partial u^{k \alpha}} .
$$

PROOF: Let $\mathrm{X}$ be a vector field defined on the vector bundle $\square$ with form (3.1).and $\mathrm{f}$ be a function defined on the vector bundle $\pi$. Then by considering Proposition 2.1. and 2.2. we write

$$
\begin{gathered}
(\mathrm{X}[\mathrm{f}]) \frac{V^{k}}{k}=\left\{\mathrm{X}^{0 \mathrm{i}} \frac{\partial f}{\partial x^{0 i}}+\mathrm{X}^{0 \alpha} \frac{\partial f}{\partial u^{0 \alpha}}\right\}_{k}^{V^{k}}=\left(\mathrm{X}^{0 \mathrm{i}}\right) \frac{V_{k}^{k}}{\partial x^{k i}}+\left(\mathrm{X}^{0 \alpha}\right) \frac{V_{k}^{k}}{\frac{\partial f_{k}^{C^{k}}}{\partial u^{k \alpha}}}
\end{gathered}
$$

If the local expression of the vertical lift $\mathrm{X}_{k}^{V^{k}}$ of $\mathrm{k}$ of $\mathrm{X}$ is

$$
\mathrm{X}_{k}^{V^{k}}=\mathrm{X}^{\mathrm{ri}} \frac{\partial}{\partial x^{r i}}+\mathrm{X}^{\mathrm{r} \alpha} \frac{\partial}{\partial u^{r \alpha}}
$$

with respect to adapted coordinates $\left\{\mathrm{x}^{\mathrm{ri}}, \mathrm{u}^{\mathrm{r} \alpha}: 0 \leq \mathrm{r} \leq \mathrm{k}\right\}$ in $\mathrm{k}_{\mathrm{E}}$. Then we write

$$
\mathrm{X}_{k}^{V^{k}}\left[\mathrm{f}_{k}^{C^{k}}\right]=\mathrm{X}^{\mathrm{ri}} \frac{\partial f_{k}^{C^{k}}}{\partial x^{r i}}+\mathrm{X}^{\mathrm{r} \alpha} \frac{\partial f_{k}^{C^{k}}}{\partial u^{r \alpha}}
$$

for all the function $\mathrm{f}$. Thus if we equalise the equation (3.3) with the equation (3.5) by considering the equation (3.2) then we obtaine the below equations

$\mathrm{X}^{\mathrm{ri}}=0, \mathrm{X}^{\mathrm{r} \alpha}=0,0 \leq \mathrm{r} \leq \mathrm{k}-1, \mathrm{X}^{\mathrm{ki}}=\left(\mathrm{X}^{0 \mathrm{i}}\right) \frac{V_{k}^{k}}{,}, \mathrm{X}^{\mathrm{k} \alpha}$

$$
=\left(\mathrm{X}^{0 \alpha}\right) \frac{V_{k}^{k}}{\text {. }} \text {. }
$$

Finally, the local expression of the vertical lift $\mathrm{X}_{k}^{V^{k}}$ of order

$\mathrm{k}$ of $\mathrm{X}$ to the extended vector bundle $\pi^{\mathrm{k}}$ is

$X_{k}^{V^{k}}=\left(X^{o i}\right)_{k}^{V^{k}} \frac{\partial}{\partial x^{k i}}+\left(X^{o \alpha}\right)_{k}^{V^{k}} \frac{\partial}{\partial u^{k \alpha}}$.

Furthermore, we define the complete lift of order $k$ of a vector field $\mathrm{X}$ defined on the vector bundle $\pi$ to its the extended vector bundle $\pi^{\mathrm{k}}=\left({ }^{\mathrm{k}} \mathrm{E}, \pi^{\mathrm{k}},{ }^{\mathrm{k}} \mathrm{M}\right)$ by using the induction method for integer number $\mathrm{k}$, then:

DEFINITION 3.2. Let $X$ be a vector field defined on the vector bundle $\square$. In that case; the complete lift of order $\mathbf{k}$ of $\mathrm{X}$ to the extended vector bundle $\pi^{\mathrm{k}}$ is a vector field defined on $\pi^{\mathrm{k}}$ which it is defined with the below equation

$$
\mathrm{X}_{k}^{C^{k}}\left(\mathrm{f}_{k}^{C^{k}}\right)=(\mathrm{X}[\mathrm{f}])_{k}^{C^{k}} ; \quad \forall \mathrm{f} \in \mathfrak{I}_{0}^{0}(\square)
$$

Now we give the below theorem connected with the components of the complete lift of order $\mathrm{k}$ of $\mathrm{X}$.

PROPOSITION 3.2. Let $X$ be a vector field defined on the vector bundle $\square$ with form (3.1). In that case; the local expression of the complete lift $\mathrm{X}_{k}^{C^{k}}$ of order $\mathrm{k}$ of $\mathrm{X}$ to the extended vector bundle $\pi^{\mathrm{k}}$ is

$$
\begin{gathered}
\mathrm{X}_{k}^{C^{k}}=\sum_{r=0}^{k}\{ \\
\left.\left(\begin{array}{l}
k \\
r
\end{array}\right)\left(X^{o i}\right)_{k}^{C^{r} V^{k-r}} \frac{\partial}{\partial x^{r i}}+\left(\begin{array}{l}
k \\
r
\end{array}\right)\left(X^{o \alpha}\right)_{k}^{C^{r} V^{k-r}} \frac{\partial}{\partial u^{r \alpha}}\right\} .
\end{gathered}
$$

PROOF: Let $X$ be a vector field defined on the vector bundle $\square$ with form (3.1).and $\mathrm{f}$ be a function defined on the vector bundle $\pi$. Then by considering Proposition 2.2. we write

$$
(\mathrm{X}[\mathrm{f}])_{k}^{C^{k}}=\left\{\mathrm{X}^{0 \mathrm{i}} \frac{\partial f}{\partial x^{0 i}}+\mathrm{X}^{0 \alpha} \frac{\partial f}{\partial u^{0 \alpha}}\right\}_{k}^{C^{k}}
$$

$=\sum_{r=0}^{k}\left({ }_{r}^{k}\right)\left(X^{0 i}\right)_{k}^{C^{r} V^{k-r}} \frac{\partial f_{k}^{C^{k}}}{\partial x^{r i}}+\left(\begin{array}{l}k \\ r\end{array}\right)\left(X^{0 \alpha}\right)_{k}^{C^{r} V^{k-r}} \frac{\partial f_{k}^{C^{k}}}{\partial u^{r \alpha}}$

If the local expression of the complete lift $\mathrm{X}_{k}^{C^{k}}$ of $\mathrm{k}$ of $\mathrm{X}$ is 


$$
\mathrm{X}_{k}^{C^{k}}=\mathrm{X}^{\mathrm{ri}} \frac{\partial}{\partial x^{r i}}+\mathrm{X}^{\mathrm{r} \alpha} \frac{\partial}{\partial u^{r \alpha}}
$$

with respect to adapted coordinates $\left\{\mathrm{x}^{\mathrm{ri}}, \mathrm{u}^{\mathrm{r} \alpha}: 0 \leq \mathrm{r} \leq \mathrm{k}\right\}$ in $\mathrm{k}_{\mathrm{E}}$. Then we write

$$
\mathrm{X}_{k}^{C^{k}}\left[\mathrm{f}_{k}^{C^{k}}\right]=\sum_{r=0}^{k} \mathrm{X}^{\mathrm{ri}} \frac{\partial f_{k}^{C^{k}}}{\partial x^{r i}}+\mathrm{X}^{\mathrm{r} \alpha} \frac{\partial f_{k}^{C^{k}}}{\partial u^{r \alpha}}
$$

for all the function $\mathrm{f}$. Thus if we equalise the equation (3.7) with the equation (3.9) by considering the equation (3.6) then we obtaine the below equations

$$
\mathrm{X}^{\mathrm{ri}}=\left(\begin{array}{c}
k \\
r
\end{array}\right)\left(\mathrm{X}^{0 \mathrm{i}}\right)_{k}^{C^{r} V^{k-r}} \underset{0 \leq \mathrm{r} \leq \mathrm{k} .}{,} \mathrm{X}^{\mathrm{r} \alpha}=\left(\begin{array}{l}
k \\
r
\end{array}\right)\left(\mathrm{X}^{0 \alpha}\right)_{k}^{C^{r} V^{k-r}}
$$

Finally, the local expression of the complete lift $\mathrm{X}_{k} C^{k}$ of order k of X to the extended vector bundle $\pi^{\mathrm{k}}$ is

$$
\begin{gathered}
\mathrm{X}_{k}^{C^{k}}=\sum_{r=0}^{k}\{ \\
\left.\left(\begin{array}{l}
k \\
r
\end{array}\right)\left(X^{o i}\right)_{k}^{C^{r} V^{k-r}} \frac{\partial}{\partial x^{r i}}+\left(\begin{array}{l}
k \\
r
\end{array}\right)\left(X^{o \alpha}\right)_{k}^{C^{r} V^{k-r}} \frac{\partial}{\partial u^{r \alpha}}\right\} .
\end{gathered}
$$

In addition to the complete-vertical lift of order $(\mathrm{r}, \mathrm{s})$ of a vector field $X$ defined on the vector bundle $\square$ to its the extended vector bundle $\square^{\mathrm{k}}$ can be defined for the integer numbers $0 \leq \mathrm{r} \leq \mathrm{k}$ and $0 \leq \mathrm{s} \leq \mathrm{k}-\mathrm{r}$.

DEFINITION 3.3. Let $X$ be a vector field defined on the vector bundle $\square$. In that case; the completevertical lift of order $(\mathbf{r}, \mathbf{s})$ of $\mathrm{X}$ to the extended vector bundle $\pi^{\mathrm{k}}$ is a vector field defined on $\pi^{\mathrm{k}}$ which it is defined with the below equation

$$
\mathrm{X}_{k}^{C^{r} V^{s}}\left(\mathrm{f}_{k}^{C^{k}}\right)=(\mathrm{X}[\mathrm{f}])_{k}^{C^{r} V^{s}} ; \quad \forall \mathrm{f} \in \mathfrak{I}_{0}{ }^{0}(\square) .
$$

Now we give the below theorem connected with the components of the complete-vertical lift of order $(r, s)$ of X.

PROPOSITION 3.3. Let $X$ be a vector field defined on the vector bundle $\square$ with form (3.1). In that case; the local expression of the complete lift $\mathrm{X}_{k}^{C^{r} V^{s}}$ of $\operatorname{order}(\mathrm{r}, \mathrm{s})$ of $\mathrm{X}$ to the extended vector bundle $\pi^{\mathrm{k}}$ is

$$
\begin{gathered}
\mathrm{X}_{k}^{C^{r} V^{s}}=\sum_{t=0}^{k}\left\{\left(\begin{array}{l}
r \\
k-t
\end{array}\right)\left(\mathrm{X}^{0 \mathrm{i}}\right)_{k}^{C^{t-s} V^{s+k-t}} \frac{\partial}{\partial x^{t i}}+\right. \\
\left.\left(\begin{array}{l}
r \\
k-t
\end{array}\right)\left(\mathrm{X}^{0 \alpha}\right)_{k}^{C^{t-s} V^{s+k-t}} \frac{\partial}{\partial u^{t \alpha}}\right\} .
\end{gathered}
$$

PROOF: Let $X$ be a vector field defined on the vector bundle $\square$ with form (3.1).and $\mathrm{f}$ be a function defined on the vector bundle $\pi$. Then by considering Proposition 2.2. we write

$$
\begin{gathered}
(\mathrm{X}[\mathrm{f}]) C_{k}^{C^{r} V^{s}}=\left\{\mathrm{X}^{0 \mathrm{i}} \frac{\partial f}{\partial x^{0 i}}+\mathrm{X}^{0 \alpha} \frac{\partial f}{\partial u^{0 \alpha}}\right\}_{k}^{C^{r} V^{s}}(3.11) \\
=\sum_{t=0}^{r}\{ \\
\left.\left(\begin{array}{c}
r \\
t
\end{array}\right)\left(X^{0 i}\right)_{k}^{C^{r-t} V^{s+t}} \frac{\partial f_{k}^{C^{k}}}{\partial x^{k-t i}}+\left(\begin{array}{c}
r \\
t
\end{array}\right)\left(X^{0 \alpha}\right)_{k}^{C^{r-t} V^{s+t}} \frac{\partial f_{k}^{C^{k}}}{\partial u^{k-t \alpha}}\right\}
\end{gathered}
$$

If the local expression of the complete-vertical lift $\mathrm{X}$ $C^{r} V^{s}$ of $\mathrm{k}$ of $\mathrm{X}$ is

$$
\mathrm{X}_{k}^{C^{r} V^{s}}=\mathrm{X}^{\mathrm{ti}} \frac{\partial}{\partial x^{t i}}+\mathrm{X}^{\mathrm{t} \alpha} \frac{\partial}{\partial u^{t \alpha}}
$$

with respect to adapted coordinates $\left\{\mathrm{x}^{\mathrm{ri}}, \mathrm{u}^{\mathrm{r} \alpha}: 0 \leq \mathrm{r} \leq \mathrm{k}\right\}$ in ${ }^{k}$. Then we write

$$
\mathrm{X}_{k}^{C^{r} V^{s}}\left[\mathrm{f}_{k}^{C^{k}}\right]=\sum_{t=0}^{k}\left\{\mathrm{X}^{\mathrm{ti}} \frac{\partial f_{k}^{C^{k}}}{\partial x^{t i}}+\mathrm{X}^{\mathrm{t} \alpha} \frac{\partial f_{k}^{C^{k}}}{\partial u^{t \alpha}}\right.
$$

for all the function $\mathrm{f}$. Thus if we equalise the equation (3.11) with the equation (3.13) by considering the equation (3.10) then we obtaine the below equations

$$
\begin{gathered}
\mathrm{X}^{\mathrm{ti}}=\left(\begin{array}{l}
r \\
k-t
\end{array}\right)\left(\mathrm{X}^{0 \mathrm{i}}\right)_{k}^{C^{t-s} V^{s+k-t}}, \mathrm{X}^{\mathrm{t} \alpha}=\left(\begin{array}{l}
r \\
k-t
\end{array}\right)\left(\mathrm{X}^{0 \alpha}\right) \\
C^{t-s} V^{s+k-t} \\
k
\end{gathered}
$$

where because $\left(\begin{array}{l}r \\ t\end{array}\right)=0$ for $\mathrm{t}>\mathrm{r}$ integer numbers $\mathrm{X}^{\mathrm{ti}}=0$ , $\mathrm{X}^{\mathrm{t} \alpha}=0$. Finally, the local expression of the complete-vertical lift $\mathrm{X}_{k}^{C^{r} V^{s}}$ of order $(\mathrm{r}, \mathrm{s})$ of $\mathrm{X}$ to the extended vector bundle $\pi^{\mathrm{k}}$ is

$$
\begin{gathered}
\mathrm{X}_{k}^{C^{r} V^{s}}=\sum_{t=0}^{k}\left\{\left(\begin{array}{l}
r \\
k-t
\end{array}\right)\left(\mathrm{X}^{0 \mathrm{i}}\right)_{k}^{C^{t-s} V^{s+k-t}} \frac{\partial}{\partial x^{t i}}+\right. \\
\left.\left(\begin{array}{l}
r \\
k-t
\end{array}\right)\left(\mathrm{X}^{0 \alpha}\right)_{k}^{C^{t-s} V^{s+k-t}} \frac{\partial}{\partial u^{t \alpha}}\right\} .
\end{gathered}
$$

PROPOSITION 3.4. For all vector fields $X, Y \in \mathfrak{I}$ $0^{1}(\pi)$ and all function $\mathrm{f} \in \mathfrak{I}_{0}{ }^{0}(\pi)$

i) $(\mathrm{X}+\mathrm{Y}){ }_{k}^{V^{k}}=\mathrm{X}_{k}^{V^{k}}+\mathrm{Y}_{k}^{V^{k}}$ 
ii) $(\mathrm{X}+\mathrm{Y}){ }_{k}^{C^{k}}=\mathrm{X}_{k}^{C^{k}}+\mathrm{Y}_{k}^{C^{k}}$

iii) (fX) $\frac{V^{k}}{k}=\mathrm{f}_{k}^{V^{k}} \mathrm{X}_{k}^{V^{k}}$

iv) (fX) ${ }_{k}^{C^{k}}=\sum_{r=0}^{k}\left(\begin{array}{l}k \\ r\end{array}\right) \mathrm{f}_{k}^{V^{k-r} C^{r}} \mathrm{X}_{k}^{C^{k-r} V^{r}}$

v) $(\mathrm{fX}){ }_{k}^{C^{r} V^{s}}=\sum_{h=0}^{r}\left(\begin{array}{l}r \\ h\end{array}\right) \mathrm{f}_{k}^{V^{s+h} C^{r-h}} \mathrm{X}_{k}^{C^{h} V^{k-h}} 0 \leq$

$\mathrm{r}, \mathrm{s} \leq \mathrm{k}(\mathrm{r}+\mathrm{s}=\mathrm{k})$.

\section{THE LIFTS OF 1-FORMS}

Let $\omega$ be a 1 -form defined on the vector bundle $\pi$ and

$$
\omega=\omega_{0 i} d x^{0 i}+\omega_{0 \alpha} d x^{0 \alpha}
$$

be local expression of $\omega$.

Thus if we define the vertical lift of a 1 -form $\omega$ defined on the vector bundle $\pi$ to its the extended vector bundle $\pi^{k}=\left({ }^{k} E, \pi^{k},{ }^{k} M\right)$ using the induction method for integer number $k$, then:

DEFINITION 4.1. Let $\omega$ be a 1 -form defined on the vector bundle $\pi$. In that case; the vertical lift of order $\boldsymbol{k}$ of $\omega$ to the extended vector bundle $\pi^{k}$ is a 1 -form defined on $\pi^{k}$ which it is defined with the below equation

$$
\omega_{k}^{V^{k}}\left(X_{k}^{C^{k}}\right)=(\omega(X))_{k}^{V^{k}} ; \quad \forall X \in \mathfrak{I}_{0}^{1}(\pi)(4.2)
$$

Now we give the below theorem connected with the components of the vertical lift of order $k$ of $\omega$.

PROPOSITION 4.1. Let $\omega$ be a 1 -form defined on the vector bundle $\pi$ with form (4.1). In that case; the local expression of the vertical lift $\omega_{k}^{V^{k}}$ of order $k$ of $\omega$ to the extended vector bundle $\pi^{k}$ is

$$
\omega_{k}^{V^{k}}=\left(\omega_{0 i}\right){ }_{k}^{V^{k}} d x^{0 i}+\left(\omega_{0} \alpha\right) V_{k}^{V^{k}} d x x^{0 \alpha} .
$$

PROOF: Let $\omega$ be a 1-form defined on the vector bundle $\pi$ with form (4.1).and $X$ be a vector field defined on the vector bundle $\pi$. Then by considering Proposition 2.1. we write

$$
\begin{gathered}
(\omega(X)){ }_{k}^{V^{k}}=\left\{\omega_{0 i} X^{0 i}+\omega_{0 \alpha} X^{0 \alpha}\right\}_{k}^{V^{k}}=\left(\omega_{0 i}\right)_{k}^{V^{k}} \\
\left(X^{0 i}\right)_{k}^{V^{k}}+\left(\omega_{0 \alpha}\right)_{k}^{V^{k}}\left(X^{0 \alpha}\right)_{k}^{V^{k}}
\end{gathered}
$$

If the local expression of the vertical lift $\omega_{k} V^{k}$ of $k$ of $\omega$ is

$$
\omega_{k}^{V^{k}}=\omega_{r i} d x^{r i}+\omega_{r \alpha} d x^{r \alpha}
$$

with respect to adapted coordinates $\left\{x^{r i}, u^{r \alpha}: 0 \leq r \leq k\right\}$ in ${ }^{k} E$. Then we write

$$
\begin{gathered}
\omega_{k}^{V^{k}}\left(X_{k}^{C^{k}}\right)=\omega_{r i}\left(\begin{array}{l}
k \\
r
\end{array}\right)\left(X^{0 i}\right){ }_{k}^{C^{r} V^{k-r}}+\omega_{r \alpha}\left(\begin{array}{l}
k \\
r
\end{array}\right)\left(X^{0 \alpha}\right. \\
{ }_{k}^{C^{r} V^{k-r}}
\end{gathered}
$$

for all the vector field $X$. Thus if we equalise the equation (4.3) with the equation (4.5) by considering the equation (4.2) then we obtaine the below equations

$$
\begin{aligned}
& \omega_{0 i}=\left(\omega_{0 i}\right)_{k}^{V^{k}}, \omega_{0 \alpha}=\left(\omega_{0 \alpha}\right)_{k}^{V^{k}}, \omega_{r i}=0, \omega_{r \alpha} \\
& =0 \quad 1 \leq r \leq k
\end{aligned}
$$

Finally, the local expression of the vertical lift $\omega_{k}^{V^{k}}$ of order $k$ of $\omega$ to the extended vector bundle $\pi^{k}$ is

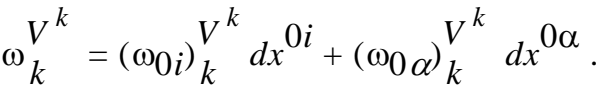

Furthermore, we define the complete lift of order $k$ of a 1 -form $\omega$ defined on the vector bundle $\pi$ to its the extended vector bundle $\pi^{k}=\left({ }^{k} E, \pi^{k},{ }_{M} M\right)$ by using the induction method for integer number $k$, then:

DEFINITION 4.2. Let $\omega$ be a 1 -form defined on the vector bundle $\pi$. In that case; the complete lift of order $k$ of $\omega$ to the extended vector bundle $\pi^{k}$ is a 1form defined on $\pi^{k}$ which it is defined with the below equation

$$
\omega_{k}^{C^{k}}\left(X_{k}^{C^{k}}\right)=(\omega(X)){ }_{k}^{C^{k}} ; \quad \forall X \in \mathfrak{I}_{0}^{1}(\pi)
$$

Now we give the below theorem connected with the components of the complete lift of order $k$ of $\omega$.

PROPOSITION 4.2. Let $\omega$ be a 1 -form defined on the vector bundle $\pi$ with form (4.1). In that case; the local expression of the complete lift $\omega_{k}^{C^{k}}$ of order $k$ of $\omega$ to the extended vector bundle $\pi^{k}$ is

$$
\begin{aligned}
& \omega_{k}^{C^{k}}=\sum_{r=0}^{k}\left\{\left(\omega_{0 i}\right){ }_{k}^{V^{r} C^{k-r}} d x^{r i}+\left(\omega_{0} \alpha\right)_{k}^{V^{r} C^{k-r}} d x^{r}\right. \\
& \left.{ }^{\alpha}\right\} \text {. }
\end{aligned}
$$


Vol. 5, Issue 12, December 2016

PROOF: Let $\omega$ be a 1 -form defined on the vector bundle $\pi$ with form (4.1). and $X$ be a vector field defined on the vector bundle $\pi$. Then by considering Proposition 2.2. we write

$$
\begin{gathered}
(\omega(X)){ }_{k}^{C^{k}}=\left\{\omega_{0 i} X^{0 i}+\omega_{0 \alpha} X^{0 \alpha}\right\}_{k}^{C^{k}}=\left\{\omega_{0 i} X^{0 i}\right\}_{k}^{C^{k}} \\
+\left\{\omega_{0 \alpha} X^{0 \alpha}\right\}_{k}^{C^{k}}(4.7) \\
=\sum_{r=0}^{k}\{ \\
\left.\left(\begin{array}{l}
k \\
r
\end{array}\right)\left(X^{0 i}\right)_{k}^{C^{r} V^{k-r}}\left(\omega_{0 i}\right)_{k}^{V^{r} C^{k-r}}+\left({ }_{r}^{k}\right)\left(X^{0 \alpha}\right)_{k}^{C^{r} V^{k-r}}\left(\omega_{o \alpha}\right)_{k}^{V^{r} C^{k-r}}\right\} .
\end{gathered}
$$

If the local expression of the complete lift $\omega_{k}^{C^{k}}$ of $k$ of $\omega$ is

$$
\omega_{k}^{C^{k}}=\omega_{r i} d x^{r i}+\omega_{r \alpha} d x^{r \alpha}
$$

with respect to adapted coordinates $\left\{x^{r i}, u^{r \alpha}: 0 \leq r \leq k\right\}$ in ${ }^{k} E$. Then we write

$$
\begin{gathered}
\omega_{k}^{C^{k}}\left(X_{k}^{C^{k}}\right)=\sum_{r=0}^{k}\{ \\
\left.\left(\begin{array}{l}
k \\
r
\end{array}\right)\left(X^{0 i}\right)_{k}^{C^{r} V^{k-r}} \omega_{r i}+\left(\begin{array}{l}
k \\
r
\end{array}\right)\left(X^{0 \alpha}\right)_{k}^{C^{r} V^{k-r}} \omega_{r \alpha}\right\}(4.9)
\end{gathered}
$$

for all the vector field $X$. Thus if we equalise the equation (4.7) with the equation (4.9) by considering the equation (4.6) then we obtain the below equations $\omega_{r i}=\left(\omega_{0 i}\right) \frac{V_{k}^{r} C^{k-r}}{,}, \omega_{r \alpha}=\left(\omega_{0 \alpha}\right)_{k}^{V^{r} C^{k-r}} \quad 0 \leq r \leq k$.

Finally, the local expression of the complete lift $\omega_{k}^{C^{k}}$ of $\omega$ to the extended vector bundle $\pi^{k}$ is

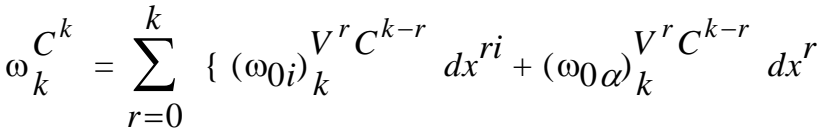

$$
\begin{aligned}
& \alpha \text {. }
\end{aligned}
$$

In addition to the complete-vertical lift of order $(r, s)$ of a 1 -form $\omega$ defined on the vector bundle $\pi$ to its the extended vector bundle $\pi^{k}$ can be defined for the integer numbers $0 \leq r \leq k$ and $0 \leq s \leq k-r$.

DEFINITION 4.3. Let $\omega$ be a 1 -form defined on the vector bundle $\pi$. In that case; the complete-vertical lift of order $(r, s)$ of $\omega$ to the extended vector bundle $\pi$ $k$ is a 1 -form defined on $\pi^{k}$ which it is defined with the below equation

$$
\omega_{k}^{C^{r} V^{s}}\left(X_{k}^{C^{k}}\right)=(\omega(X)){ }_{k}^{C^{r} V^{s}} ; \forall X \in \mathfrak{I}_{0}^{1}(\pi)(4.10)
$$

Now we give the below theorem connected with the components of the complete-vertical lift of order $(r, s)$ of $\omega$.

PROPOSITION 4.3. Let $\omega$ be a 1 -form defined on the vector bundle $\pi$ with form (4.1). In that case; the local expression of the lift $\omega_{k}^{C^{r} V^{s}}$ of $\omega$ to the extended vector bundle $\pi^{k}$ is

$$
\begin{aligned}
& \omega_{k}^{C^{r} V^{s}}=\sum_{t=0}^{k}\left\{\frac{\left(\begin{array}{l}
r \\
t
\end{array}\right)}{\left(\begin{array}{l}
k \\
t
\end{array}\right)}\left(\omega_{0 i}\right)_{k}^{V^{s+t} C^{r-t}} d x^{t i}+\frac{\left(\begin{array}{l}
r \\
t
\end{array}\right)}{\left(\begin{array}{l}
k \\
t
\end{array}\right)}\left(\omega_{r}\right.\right. \\
& \left.\alpha^{V^{s+t}} C^{r-t} d u^{r \alpha}\right\} \text {. }
\end{aligned}
$$

PROOF: Let $\omega$ be a 1 -form defined on the vector bundle $\pi$ with form (4.1).and $\omega$ be a vector field defined on the vector bundle $\pi$. Then by considering Proposition 2.2. we write

$$
\begin{aligned}
& (\omega(X)){ }_{k}^{C^{r} V^{s}}=\left\{\omega_{0 i} X^{0 i}+\omega_{0 i} X^{0 \alpha}\right\}_{k}^{C^{r} V^{s}}=\sum_{t=0}^{r}\{ \\
& \left(\begin{array}{c}
r \\
t
\end{array}\right)\left(\omega_{0 i}\right)_{k}^{C^{r-t} V^{s+t}}\left(X^{0 i}\right)_{k}^{V^{k-t} C^{t}}+\left(\begin{array}{c}
r \\
t
\end{array}\right)\left(\omega_{0 \alpha}\right)_{k}^{C^{r-t} V^{s+t}}\left(X^{0 \alpha}\right)_{k}^{V^{k-t} C^{t}} \\
& \text { (4.11) }
\end{aligned}
$$

If the local expression of the complete-vertical lift $\omega$ $C^{r} V^{s}$ of $k$ of $\omega$ is

$$
\omega_{k}^{C^{k}}=\omega_{r i} d x^{r i}+\omega_{r \alpha} d x^{r \alpha}
$$

with respect to adapted coordinates $\left\{x^{r i}, u^{r \alpha}: 0 \leq r \leq k\right\}$ in ${ }^{k} E$. Then we write

$$
\begin{gathered}
\omega_{k}^{C^{r} V^{s}}\left(X_{k}^{C^{k}}\right)=\sum_{t=0}^{k}\{ \\
\left.\left(\begin{array}{l}
k \\
t
\end{array}\right)\left(X^{0 i}\right)_{k}^{C^{t} V^{k-t}} \omega_{t i}+\left(\begin{array}{c}
k \\
t
\end{array}\right)\left(X^{0 \alpha}\right)_{k}^{C^{t} V^{k-t}} \omega_{t \alpha}\right\}(
\end{gathered}
$$

for all the vector field $X$. Thus if we equalise the equation (4.11) with the equation (4.13) by considering the equation (4.10) then we obtaine the below equations

$$
\begin{gathered}
\omega_{t i}=\frac{\left(\begin{array}{l}
r \\
t
\end{array}\right)}{\left(\begin{array}{l}
k \\
t
\end{array}\right)}\left(\omega_{0 i}\right)_{k}^{V^{s+t} C^{r-t}} \quad, \omega_{r \alpha}=\frac{\left(\begin{array}{l}
r \\
t
\end{array}\right)}{\left(\begin{array}{l}
k \\
t
\end{array}\right)}\left(\omega_{r \alpha}\right) \\
V^{s+t} C^{r-t} \quad 0 \leq t \leq k
\end{gathered}
$$


where because $\left(\begin{array}{l}r \\ t\end{array}\right)=0$ for $\mathrm{t}>\mathrm{r}$ integer numbers $\omega_{t i}=$ $0, \omega_{t \alpha}=0$. Finally, the local expression of the complete-vertical lift $\omega_{k}^{C^{r} V^{s}}$ of order $(r, s)$ of $\omega$ to the extended vector bundle $\pi^{k}$ is

$$
\begin{aligned}
& \omega_{k}^{C^{r} V^{s}}=\sum_{t=0}^{k}\left\{\frac{\left(\begin{array}{l}
r \\
t
\end{array}\right)}{\left(\begin{array}{c}
k \\
t
\end{array}\right)}\left(\omega_{0 i}\right) V_{k}^{s+t} C^{r-t} d x+\frac{\left(\begin{array}{c}
r \\
t
\end{array}\right)}{\left(\begin{array}{c}
k \\
t
\end{array}\right)}\left(\omega_{r}\right.\right. \\
& \alpha)_{k}^{\left.V^{s+t} C^{r-t} d u^{t \alpha}\right\} .}
\end{aligned}
$$

PROPOSITION 4.4. For all 1 -forms $\omega, \theta \in \mathfrak{I}_{1}{ }^{0}(\pi)$ and all function $f \in \mathfrak{I}_{0}{ }^{0}(\pi)$

i) $(\omega+\theta) \frac{V_{k}^{k}}{k}=\omega_{k}^{V^{k}}+\theta_{k}^{V^{k}}$

ii) $(\omega+\theta){ }_{k}^{C^{k}}=\omega_{k}^{C^{k}}+\theta_{k}^{C^{k}}$

iii) $(f \omega) \frac{V^{k}}{k}=f_{k}^{V^{k}} \omega_{k}^{V^{k}}$

iv) $(f \omega)_{k}^{C^{k}}=\sum_{r=0}^{k}\left(\begin{array}{l}k \\ r\end{array}\right) f_{k}^{V^{k-r} C^{r}} \omega_{k}^{C^{k-r} V^{r}}$

v) $(f \omega)_{k}^{C^{r} V^{s}}=\sum_{h=0}^{r}\left(\begin{array}{l}r \\ h\end{array}\right) f_{k}^{V^{s+h} C^{r-h}} \omega_{k}^{C^{h} V^{k-h}} 0 \leq$ $r, s \leq \mathrm{k}(r+s=k)$.

\section{CONCLUSION}

This study is the generalising study of the completevertical lift of the differential elements defined on the extended vector bundles. In addition to the previous studies connected with the vertical and complete lift of differential elements are special cases of this study.

\section{REFERENCES}

[1] Aycan C., Lifts on Jet Bundles and Prolongation Relations, M.Sc. Thesis, Pamukkale Univ., Denizli, 1998.

[2] Aycan C., The Lifts of Euler-Lagrange and Hamilton Equations on the Extended Jet Bundles, D. Sc. Thesis, Osmangazi Univ., Eski S ehir, 2003.

[3] Etoya, J.J.: On a Complete Lifting of Derivations, Tensor, 38(1982), 169-178.

[4] Etoya, J.J.: Derivations in Tangent Bundle, Differential Geometry Proceeding of The International Symposium Held at Peniscola, Spain(October 3-10, 1982), 43-52.

[5] Etoya, J.J.: Lifts of Derivations to the Tangent Bundle, Proceeding of the IV International Colloquium of Differential Geometry. Santiago de Compostela(1979), 117-130.

[6] Saunders, D.J.: The Geometry of Jet Bundles, Cambridge University Press, Cambridge, 1989.

[7] Sato, I.: Complete Lifts from a Manifold to its Cotangent Bundle, Kodai Math. Sem. Rep., 20(1968), 458-468.

[8] Tani, M.: Prolongations of Hypersurfaces to Tangent Bundles, Kodai Math. Sem. Rep., 21(1969), 85-96.
[9] Yano, K., Davies, E.T.: Metrics and Connections in Tangent Bundles, Kodai Math. Sem. Rep., 23(1971), 493-504.

[10] Yano, K., Ishihara, S.: Tangent and Cotangent Bundles, Marcel Dekker Inc., New York, 1973.

[11] Yano, K., Patterson, E.M.: Vertical Lifts from a Manifold to its Cotangent Bundle, Jour. Math. Soc. Japan., 19(1967), 91-113.

[12] Yano, K., Patterson, E.M.: Horizontal Lifts from a Manifold to its Cotangent Bundle, Jour. Math. Soc. Japan., 19(1967), 185-198. 